\title{
Statistical field theory for polar fluids
}

Bilin Zhuang, and Zhen-Gang Wang

Citation: The Journal of Chemical Physics 149, 124108 (2018); doi: 10.1063/1.5046511

View online: https://doi.org/10.1063/1.5046511

View Table of Contents: http://aip.scitation.org/toc/jcp/149/12

Published by the American Institute of Physics 


\title{
Statistical field theory for polar fluids
}

\author{
Bilin Zhuang ${ }^{1,2}$ and Zhen-Gang Wang ${ }^{1, a)}$ \\ ${ }^{1}$ Division of Chemistry and Chemical Engineering, California Institute of Technology, \\ Pasadena, California 91125, USA \\ ${ }^{2}$ Department of Materials Science and Engineering, Institute of High Performance Computing, \\ Singapore 138632, Singapore
}

(Received 28 June 2018; accepted 3 September 2018; published online 26 September 2018)

\begin{abstract}
Using a variational field-theoretic approach, we derive a theory for polar fluids. The theory naturally accounts for the reaction field without resorting to the cavity construct and leads to a simple formula for the dielectric constant in terms of the molecular dipole moment and density. We apply our formula to calculate the dielectric constants of nonpolarizable liquid models for more than a hundred small molecules without using any adjustable parameters. Our formula predicts dielectric constants of these nonpolarizable liquid models more accurately than the Onsager theory and previous field-theoretic dielectric theories, as demonstrated by the closer agreement to the simulation results. The general theory also yields the free energy, which can describe the response of polar fluids under applied electric fields. Published by AIP Publishing. https://doi.org/10.1063/1.5046511
\end{abstract}

\section{INTRODUCTION}

The dielectric constant is one of the most basic properties of fluids. It is fundamental to many aspects of science and technology, including behaviors of biological molecules, ${ }^{1,2}$ mechanisms of chemical reactions, ${ }^{3-5}$ fabrication of new materials, ${ }^{6-8}$ solubility of polar and nonpolar species, ${ }^{9,10}$ and development of energy storage devices. ${ }^{11}$ Accurate determination of the dielectric constant from molecular parameters, especially in the liquid phase, remains a challenge, whether theoretically or through computer simulations. ${ }^{11}$

Many attempts have been made. ${ }^{12,13}$ In 1912, Debye derived an equation relating the dielectric constant to the molecular permanent dipole moment and the isotropic polarizability. As Debye assumed each dipole independently interacts with the applied field, his theory did not take into account the intermolecular dipole-dipole correlations; therefore, the Debye theory is only applicable for gas-phase systems in the dilute limit. ${ }^{14,15}$ In 1936, Onsager developed a theory by considering a point dipole in a cavity that is embedded in a homogeneous dielectric continuum. This point dipole induces polarization in the surrounding medium, which gives rise to a reaction field that in turn influences the dipole alignment in the cavity. ${ }^{16}$ The Onsager theory yields a much improved prediction for liquid dielectric constants than the Debye theory, as validated by experimental measurements. ${ }^{17}$ Shortly after, Kirkwood extended Onsager's theory by introducing a $g$-factor to account for the short-range orientational correlation between the molecules. ${ }^{18}$ While Kirkwood's formulation is especially relevant for determining dielectric constants with computer simulation, ${ }^{19}$ it is often difficult to evaluate the $g$-factor analytically because

a)zgw@cheme.caltech.edu detailed information about the molecular interaction is often unavailable. ${ }^{17,20}$ Since then, more sophisticated theories for the dielectric constant have been developed based on perturbative expansions, ${ }^{21-23}$ cluster expansions, ${ }^{24-28}$ integralequation theories, ${ }^{29-34}$ and by considering the specific shapes of molecules ${ }^{35,36}$ or intermolecular bonding. ${ }^{37}$ However, the Debye equation and the Onsager equation remain popular due to their simplicity.

Despite the many attempts, there remains a need for a simple theory for calculating dielectric constants of fluids based on readily available molecular parameters. Methods based on perturbative expansions are unsatisfactory because the series often do not converge with increasing number of terms. ${ }^{38}$ Cluster expansion methods and integral-equation methods are able to relate dielectric constants to molecular properties, but the required five-dimensional correlation functions (three for positions and two for orientations) are very cumbersome to evaluate. ${ }^{28,39}$

In recent years, progress in field-theoretic statisticalmechanical methods has provided an avenue for the development of new and simple expressions for dielectric constants. An additional important advantage of the fieldtheoretic approach is that it can be conveniently integrated into a wide range of platforms for studying soft-matter and polymeric systems where the molecules are modeled with the same approach. ${ }^{40-42}$ One of the simpler field-theoretic methods is the self-consistent-field theory (SCFT), where one approximates the partition function using the saddle-point approximation. Several studies have applied this approach ${ }^{43-47}$ and the results are comparable to the predictions of the Debye theory. Beyond the saddle-point approximation, Levy, Andelman, and Orland derived an expression for the dielectric constant by considering the one-loop expansion of the free energy. ${ }^{48,49}$ More recently, Martin et al. derived the dielectric constant by pursuing a simple gaussian field approximation to the 
field-theoretic Hamiltonian. ${ }^{50}$ However, the numerical performance of the predictions by these field-theoretic theories is less than satisfactory when compared to the simulation or experimental data. In this work, we develop a field-theoretic theory for calculating dielectric constants of pure liquids using a variational approach, ${ }^{51,52}$ with the goal to improve the overall accuracy in predicting dielectric constants using only a few readily available parameters.

The dipolar renormalized gaussian fluctuation (DRGF) theory presented in this work is formulated using a field-based gaussian reference action that involves an interaction tensor and the average of the fluctuating field as the variational parameters. The self-consistency condition on the interaction tensor and the average field naturally accounts for the effects of the reaction field. While in the current work we consider nonpolarizable fluids for simplicity, effects of polarizability can be included in a straightforward way and we reserve the effort for an upcoming paper. The resulting theory consists of simple analytical expressions for the free energy and the dielectric constant. In particular, the dielectric constant of a liquid can be calculated based on the permanent dipole moment and the number concentration of the molecules, both being readily available parameters for many systems, without the need for any fitting parameters. We apply the DRGF theory to calculate the dielectric constants of nonpolarizable molecular models of a wide range of liquids, and we find the results from the DRGF theory provide better agreement with simulation data than previous field-theoretic approaches. ${ }^{4-50}$ The line of bestfit through our predicted dielectric constants vs. simulation data has a slope very close to 1 on a log-log plot, which is better than the Onsager theory.

The rest of this article is organized as follows. In Sec. II, we describe the model and formulate the exact partition function of the system in the field-theoretic representation. Then, we introduce a gaussian reference action and apply the variational approach based on the Gibbs-Feynman-Bogoliubov variational principle to derive a general theory for the free energy of dipolar liquids. We then consider a weak applied field in the linear response regime to obtain an expression for the dielectric constant of a liquid. In Sec. III, we apply our theory to calculate the dielectric constants for the GAFF (generalized Amber force field) and the OPLS/AA (all-atom optimized potential for liquid simulations) molecular models for an extensive set of over a hundred molecular liquids that have been studied in benchmark simulations by Caleman et al. ${ }^{53}$ For comparison, we also compute the dielectric constants with the Onsager equation and previous field-theoretic methods (based on the saddle-point approximation, the oneloop expansion, and the gaussian field approximation). Finally, in Sec. IV, we summarize the key features of the DRGF theory and offer some concluding remarks.

\section{FIELD-THEORETIC VARIATIONAL THEORY FOR POLAR LIQUIDS}

\section{A. The model}

We consider a system of $N$ polar molecules at uniform density $\rho$ in the presence of an applied field $\mathbf{E}_{0}(\mathbf{r})$. The polar molecules are modeled to be nonpolarizable and each molecule has a permanent dipole moment of magnitude $\bar{\mu}$. The microscopic state of the system can be specified by the set of positions and dipole vectors of all molecules $\left\{\left(\mathbf{r}_{i}, \boldsymbol{\mu}_{i}\right)\right.$ : $i=1,2, \ldots, N\}$, where $\mathbf{r}_{i}$ and $\boldsymbol{\mu}_{i}$ denote, respectively, the position and the dipole vector (where $\left|\mu_{i}\right|=\bar{\mu}$ ) of the $i$ th molecule. The electrostatic interaction energy $U$ of the system is the sum of all pairwise dipole-dipole interactions and the interaction energy with the applied field,

$$
U=\frac{1}{2} \sum_{i=1}^{N} \sum_{\substack{j=1 \\ i \neq j}}^{N} \boldsymbol{\mu}_{i} \mathbf{T}\left(\mathbf{r}_{i}-\mathbf{r}_{j}\right) \boldsymbol{\mu}_{j}-\sum_{i=1}^{N} \boldsymbol{\mu}_{i} \cdot \mathbf{E}_{0}\left(\mathbf{r}_{i}\right),
$$

where $\mathbf{T}(\mathbf{r})=-\nabla \nabla\left(1 / 4 \pi \varepsilon_{0}|\mathbf{r}|\right)$ is the dipole-dipole interaction tensor, with $\varepsilon_{0}$ being the vacuum permittivity.

Mathematically, improper integrals involving $\mathbf{T}(\mathbf{r})$ are nonunique and lead to a problem of "conditional convergence." However, the problem is well understood ${ }^{54,55}$ and a consistent interpretation is obtained by representing $\mathbf{T}(\mathbf{r})$ in the following form: ${ }^{56}$

$$
\begin{aligned}
\mathbf{T}(\mathbf{r}) & =-H(|\mathbf{r}|-\eta) \nabla \nabla \frac{1}{4 \pi \varepsilon_{0}|\mathbf{r}|}+\frac{1}{3 \varepsilon_{0}} \mathbb{1} \delta(\mathbf{r}) \\
& =H(|\mathbf{r}|-\eta)\left(\frac{1}{4 \pi \varepsilon_{0}|\mathbf{r}|^{3}}\left[\mathbb{1}-\frac{3 \mathbf{r r}}{|\mathbf{r}|^{2}}\right]\right)+\frac{1}{3 \varepsilon_{0}} \mathbb{1} \delta(\mathbf{r}),
\end{aligned}
$$

where $\mathbb{1}$ is the unit dyadic, $\delta(\mathbf{r})$ is the Dirac delta function, and $H(x)$ is the Heaviside step function. $\eta$ is a regularization parameter which will eventually be taken to be 0 in the final calculations. Since both terms in $\mathbf{T}(\mathbf{r})$ are well defined, it is now straightforward to compute the Fourier transform of $\mathbf{T}(\mathbf{r})$ and the result is ${ }^{39,56}$

$$
\widetilde{\mathbf{T}}(\mathbf{k})=\frac{\mathbf{k} \mathbf{k}}{\varepsilon_{0} k^{2}},
$$

where a tilde above a quantity denotes the Fourier transform $\tilde{f}(\mathbf{k})=\int d \mathbf{r} f(\mathbf{r}) e^{-i \mathbf{k} \cdot \mathbf{r}}$. Noting that $\mathbf{k k} / k^{2}$ is a projection operator, we have the following relation:

$$
\left(\varepsilon_{0} \widetilde{\mathbf{T}}(\mathbf{k})\right)^{n}=\varepsilon_{0} \widetilde{\mathbf{T}}(\mathbf{k}),
$$

which turns out to be useful in the algebraic manipulations. In addition, $\widetilde{\mathbf{T}}(\mathbf{k}=\mathbf{0})=\mathbb{1} / 3 \varepsilon_{0}$ follows from Eq. (2).

For convenience, we develop the statistical mechanics for the polar liquid in a grand canonical ensemble of the system under chemical potential $\mu$, inverse temperature $\beta=1 / k_{B} T$, and volume $V$. The grand partition function of the system is then given by

$$
\Xi=\sum_{N=0}^{\infty} \frac{e^{\beta \mu N}}{N !} Z(N),
$$

where $Z(N)$ is the canonical partition function for a system of $N$ particles, in which the Boltzmann factor is integrated over the $N$-particle configuration space as

$$
Z(N)=\frac{1}{\Lambda^{3 N}} \prod_{i} \int d \mathbf{r}_{i} \int \frac{d \Omega_{i}}{4 \pi} e^{-\beta U} .
$$

In the above expression, $\Lambda$ is the thermal de Broglie wavelength and the integral over the dipole moment is carried 
out over the solid angle $\Omega_{i}$ of the permanent dipole. The full interaction energy in general includes nonelectrostatic interactions between the particles. These interactions can be included explicitly in the field theoretical model, ${ }^{42}$ or more simply by imposing the condition of local incompressibility, in a coarsegrained description of the excluded volume effects. ${ }^{57}$ Since these nonelectrostatic interactions do not introduce significant fluctuation effects in a homogeneous bulk liquid, they may be treated at the mean-field level. These mean-field contributions can be readily included in the overall free energy. In the present work, we do not explicitly account for these nonelectrostatic interactions in the formulation of the theory in order to leave freedom for the different forms and treatments of these interactions. From Eq. (5), the grand potential $W$ of the system is given by

$$
\beta W=-\ln \Xi
$$

To move toward a continuum field description, we introduce the microscopic polarization $\hat{\mathbf{P}}(\mathbf{r})$,

$$
\hat{\mathbf{P}}(\mathbf{r})=\sum_{i=1}^{N} \boldsymbol{\mu}_{i} \delta\left(\mathbf{r}-\mathbf{r}_{i}\right)
$$

so the energy $U$ may now be rewritten as

$U=\frac{1}{2} \int d \mathbf{r} \int d \mathbf{r}^{\prime} \hat{\mathbf{P}}(\mathbf{r}) \mathbf{T}\left(\mathbf{r}-\mathbf{r}^{\prime}\right) \hat{\mathbf{P}}\left(\mathbf{r}^{\prime}\right)-\int d \mathbf{r} \hat{\mathbf{P}}(\mathbf{r}) \cdot \mathbf{E}_{0}(\mathbf{r})$.

The first term in Eq. (9) now includes self-interaction of the form $\frac{1}{2} \boldsymbol{\mu}_{i} \mathbf{T}\left(\mathbf{r}_{i}-\mathbf{r}_{i}\right) \boldsymbol{\mu}_{i}$ which is divergent for point dipoles. Such a divergence can be avoided by introducing a distribution of finite spatial width as in Ref. 51 or by imposing a momentum cutoff in the Fourier integral; we choose the latter.

The pairwise particle-particle interactions entail enormous degrees of freedom in the configuration space, making evaluation of the configurational integral intractable. To decouple the pairwise interactions, we employ field-theoretic techniques to transform the pairwise interactions into interactions between each particle and a fluctuating field. ${ }^{42}$ Here, since the dipole-dipole interaction tensor $\mathbf{T}(\mathbf{r})$ does not have an inverse operator, we perform the transformation using the Faddeev-Popov method. ${ }^{40,42,58}$ The resulting partition function becomes one involving quadratic interactions between the field variables and a one-body interaction between the particles and the auxiliary field. Upon integration over the molecular degrees of freedom, the resulting grand partition function is written as functional integrals over the polarization and the auxiliary fluctuating field. The transformation is formally exact; we defer the details of the transformation to Appendix A. Here, we simply write down the resulting field-based grand partition function as

$$
\Xi=\int \mathcal{D} \mathbf{P} \int \mathcal{D} \mathcal{G} e^{-L[\mathbf{P}, \mathcal{G}]},
$$

where the field-theoretic action $L$ is given by

$$
\begin{aligned}
L[\mathbf{P}, \mathcal{G}]= & \frac{1}{2} \int d \mathbf{r} \int d \mathbf{r}^{\prime} \mathbf{P}(\mathbf{r}) \mathcal{T}\left(\mathbf{r}-\mathbf{r}^{\prime}\right) \mathbf{P}\left(\mathbf{r}^{\prime}\right) \\
& +i \int \mathbf{P}(\mathbf{r}) \cdot \mathcal{G}(\mathbf{r})-\int \mathbf{P}(\mathbf{r}) \cdot \mathcal{E}_{0}(\mathbf{r}) \\
& -\lambda \int d \mathbf{r} \int d \Omega e^{i \boldsymbol{\mu} \cdot \mathcal{G}(\mathbf{r})} .
\end{aligned}
$$

In the above expression, we have defined $\mathcal{T}=\beta \mathbf{T}$ and $\mathcal{E}_{0}=\beta \mathbf{E}_{0}$ for simplicity of notation. $\lambda=e^{\beta \mu} /\left(4 \pi \Lambda^{3}\right)$ is the fugacity of the molecule scaled by the inverse temperature. The last term in $L$, which arises from the single-molecule partition function in the auxiliary field $i \mathcal{G}$, makes the integral for $\Xi$ non-gaussian. In Sec. II B, we introduce an approximation scheme for the field-based partition function and the grand potential, from which we derive other thermodynamic properties.

\section{B. The variational approximation}

The non-gaussian nature of the exact field-theoretic partition function $\Xi$ makes it impossible to evaluate the functional integrals exactly. A popular approximation is the selfconsistent-field theory, in which one simply approximates the partition function by taking the saddle-point value of the field-theoretic action with respect to the functional arguments. However, the self-consistent-field approach does not take into account fluctuations in the polarization and the auxiliary fluctuating field and as a result cannot capture the reaction field effects. In this section, we apply a variational approach to provide an approximate treatment of the nonlinear fluctuation effects that are responsible for the reaction field.

We begin by introducing a reference gaussian action $L_{0}$ as an approximate description for our system,

$$
\begin{aligned}
L_{0}[\mathbf{P}, \mathcal{G}]= & \frac{1}{2} \int d \mathbf{r} \int d \mathbf{r}^{\prime} \mathbf{P}(\mathbf{r}) \mathcal{T}\left(\mathbf{r}-\mathbf{r}^{\prime}\right) \mathbf{P}\left(\mathbf{r}^{\prime}\right)+i \int \mathbf{P}(\mathbf{r}) \cdot \mathcal{G}(\mathbf{r}) \\
& -\int \mathbf{P}(\mathbf{r}) \cdot \mathcal{E}_{0}(\mathbf{r})-\frac{1}{2} \int d \mathbf{r} \int d \mathbf{r}^{\prime}[i \mathcal{G}(\mathbf{r})-\mathcal{F}(\mathbf{r})] \\
& \times \mathcal{A}^{-1}\left(\mathbf{r}-\mathbf{r}^{\prime}\right)\left[i \mathcal{G}\left(\mathbf{r}^{\prime}\right)-\mathcal{F}\left(\mathbf{r}^{\prime}\right)\right]
\end{aligned}
$$

where the field-interaction tensor $\mathcal{A}(\mathbf{r})$ and the average fluctuating field $\mathcal{F}$ are the variational parameters to be determined below. The inverse of the operator $\mathcal{A}^{-1}$ is defined via $\int d \mathbf{r}^{\prime} \mathcal{A}\left(\mathbf{r}-\mathbf{r}^{\prime}\right) \mathcal{A}^{-1}\left(\mathbf{r}^{\prime}-\mathbf{r}^{\prime \prime}\right)=\mathbb{1} \delta\left(\mathbf{r}-\mathbf{r}^{\prime \prime}\right)$. In the absence of the external field $\mathcal{E}_{0}$, the field-interaction tensor $\mathcal{A}$ is isotropic. Although it is possible to account for the anisotropy of $\mathcal{A}$ for arbitrarily large $\mathcal{E}_{0}$, in this work, we are concerned with the weak field limits. Therefore, for simplicity of the theory, $\mathcal{A}(\mathbf{r})$ is assumed to be isotropic such that $\mathcal{A}(\mathbf{r})=a(\mathbf{r}) \mathbb{1}$, where $a(\mathbf{r})$ now is the scalar variational parameter that describes the field-interaction strength.

The exact field-theoretic action $L$ and the reference gaussian action $L_{0}$ only differ in their respective last terms. Essentially, the interaction between a single molecule and the auxiliary field in the exact action $L$ is replaced by a quadratic interaction of the auxiliary field in the approximate action $L_{0}$. This quadratic interaction makes the overall form of the partition function gaussian, with renormalized variational parameters $\mathcal{A}(\mathbf{r})$ and $\mathcal{F}$ that are determined self-consistently. Therefore, we shall refer to our theory as the dipolar renormalized gaussian fluctuation (DRGF) theory.

Using the reference action, we can obtain an approximation of $W$ based on the Gibbs-Feynman-Bogoliubov inequality 59 


$$
\beta W \leq-\ln \Xi_{0}+\left\langle L-L_{0}\right\rangle_{0},
$$

where $\Xi_{0}$ is the reference partition function given by

$$
\Xi_{0}=\int \mathcal{D} \mathbf{P} \int \mathcal{D} \mathcal{G} e^{-L_{0}[\mathbf{P}, \mathcal{G}]}
$$

and $\langle\mathcal{O}\rangle_{0}$ is the average of an observable $\mathcal{O}$ evaluated in the reference ensemble, i.e.,

$$
\langle\mathcal{O}\rangle_{0}=\frac{1}{\Xi_{0}} \int \mathcal{D} \mathbf{P} \int \mathcal{D} \mathcal{G} \mathcal{O}[\mathbf{P}, \mathcal{G}] e^{-L_{0}[\mathbf{P}, \mathcal{G}]}
$$

Since we will approximate $\beta W$ by the stationary value of the right-hand side in Eq. (13), ${ }^{60,79}$ for economy of notation, we will use $W$ to denote the variational free energy, with the understanding that only its stationary value corresponds (approximately) to the equilibrium free energy. The evaluation of $W$ involves performing a series of gaussian functional integrals, the details of which we defer to Appendix B. The resulting expression for $W$ is

$$
\begin{aligned}
\beta W= & -\frac{1}{2} V \int \frac{d \mathbf{k}}{(2 \pi)^{3}}\left[\ln \left(\frac{\varepsilon_{0} \tilde{a}(\mathbf{k})}{\beta+\varepsilon_{0} \tilde{a}(\mathbf{k})}\right)+\frac{\beta}{\beta+\varepsilon_{0} \tilde{a}(\mathbf{k})}\right] \\
& -\frac{1}{2} \int \frac{d \mathbf{k}}{(2 \pi)^{3}}\left(\frac{\varepsilon_{0}}{\beta+\varepsilon_{0} \tilde{a}(\mathbf{k})}\right)^{2}\left[\widetilde{\mathcal{E}}_{0}(\mathbf{k})-\widetilde{\mathcal{F}}(\mathbf{k})\right] \\
& \times \widetilde{\mathcal{T}}(\mathbf{k})\left[\widetilde{\mathcal{E}}_{0}(-\mathbf{k})-\widetilde{\mathcal{F}}(-\mathbf{k})\right]-\lambda \int d \mathbf{r} \int d \Omega e^{-f(\boldsymbol{\mu}, \mathbf{r})} .
\end{aligned}
$$

The last term in $\beta W$ can be regarded as the renormalized single-particle partition function, with $f(\boldsymbol{\mu}, \mathbf{r})$ being the effective single-particle potential given by

$$
f(\boldsymbol{\mu}, \mathbf{r})=\frac{1}{2} \boldsymbol{\mu} \cdot \mathcal{T}_{R} \cdot \boldsymbol{\mu}-\boldsymbol{\gamma}(\mathbf{r}) \cdot \boldsymbol{\mu},
$$

where

$$
\begin{aligned}
\mathcal{T}_{R} & =\int \frac{d \mathbf{k}}{(2 \pi)^{3}} \frac{\varepsilon_{0} \tilde{a}(\mathbf{k})}{\beta+\varepsilon_{0} \tilde{a}(\mathbf{k})} \widetilde{\mathcal{T}}(\mathbf{k}) \\
& =\mathcal{T}(\mathbf{0})-\int \frac{d \mathbf{k}}{(2 \pi)^{3}} \frac{\beta}{\beta+\varepsilon_{0} \tilde{a}(\mathbf{k})} \widetilde{\mathcal{T}}(\mathbf{k})
\end{aligned}
$$

with $\mathcal{T}(\mathbf{0})=\int \frac{d \mathbf{k}}{(2 \pi)^{3}} \widetilde{\mathcal{T}}(\mathbf{k})$ and

$$
\tilde{\gamma}(\mathbf{k})=\widetilde{\mathcal{E}}_{0}(\mathbf{k})-\frac{\varepsilon_{0}}{\beta+\varepsilon_{0} \tilde{a}(\mathbf{k})} \widetilde{\mathcal{T}}(\mathbf{k})\left[\widetilde{\mathcal{E}}_{0}(\mathbf{k})-\widetilde{\mathcal{F}}(\mathbf{k})\right] .
$$

We determine the stationary condition for the free energy Eq. (16) by setting $\partial W / \partial \tilde{a}(\mathbf{k})=0$ and $\partial W / \partial \widetilde{\mathcal{F}}(\mathbf{k})=\mathbf{0}$. Upon minor simplification of the functional derivatives (with details provided in Appendix C), we obtain the following two constitutive relations, which are to be solved simultaneously to determine the variational parameters:

$$
\frac{\beta V}{\varepsilon_{0} \tilde{a}(\mathbf{k})}=\lambda \int d \mathbf{r} \int d \Omega \boldsymbol{\mu} \cdot \widetilde{\mathcal{T}}(\mathbf{k}) \cdot \boldsymbol{\mu} e^{-f(\boldsymbol{\mu}, \mathbf{r})}
$$

and

$\left(\frac{\varepsilon_{0}}{\beta+\varepsilon_{0} \tilde{a}(\mathbf{k})}\right)\left[\widetilde{\mathcal{E}}_{0}(\mathbf{k})-\widetilde{\mathcal{F}}(\mathbf{k})\right]=\lambda \int d \mathbf{r} \int d \Omega \boldsymbol{\mu} e^{-f(\boldsymbol{\mu}, \mathbf{r})-i \mathbf{k} \cdot \mathbf{r}}$.

In addition, the fugacity $\lambda$ can be related to the fluid density using the statistical mechanics relation $\rho V=\langle N\rangle=-\partial W / \partial \mu$ as

$$
\rho V=\lambda \int d \mathbf{r} \int d \Omega \exp [-f(\boldsymbol{\mu}, \mathbf{r})]
$$

The effective single-particle potential $f(\boldsymbol{\mu}, \mathbf{r})$ in Eq. (17) describes how a dipole interacts with the polar environment and the applied field. The first term in $f(\boldsymbol{\mu}, \mathbf{r})$ describes the selfinteraction of a dipole in the effective field, with the effective self-interaction strength quantified by the tensor $\mathcal{T}_{R}$. Physically, this effective self-interaction includes the effects of the reaction field in condensed polar liquids - as a dipole exerts a field on its surroundings, the surroundings exert a field back on the dipole itself. From Eq. (20a), in the dilute limit $(\lambda \rightarrow 0)$, $\tilde{a}(\mathbf{k}) \rightarrow \infty$, so the reaction field vanishes and the self-energy term becomes $\frac{1}{2} \boldsymbol{\mu} \mathcal{T}(\mathbf{0}) \boldsymbol{\mu}$, which is precisely the self-energy of an isolated dipole moment. The second term in $f(\boldsymbol{\mu}, \mathbf{r})$ describes the interaction of a dipole with an effective local directing field $\gamma(\mathbf{r})$ on the dipole.

The constitutive relations Eqs. (20a) and (20b) are to be solved simultaneously for the variational parameters $a$ and $\mathcal{F}$, and these relations also allow us to infer physics of polar fluids. From Eq. (20a), we observe that the stationary value of the field-interaction parameter $a$ is related to the averaged selfinteraction under the effective single-particle potential. As we have alluded to earlier, this self-interaction is a manifestation of the reaction field; see Sec. II C 3 for further discussion. Then, from Eq. (20b), we see that the average auxiliary field $\mathcal{F}$ is a combination of the applied field $\mathcal{E}_{0}$ and the angularly averaged dipole moment in the effective single-particle potential. This combination suggests that the auxiliary field fluctuates around an averaged value that is dependent on both the applied field and the electric field generated by the dipolar material.

The variational grand potential $W$ in Eq. (16) is the centerpiece in our statistical field theory, from which all other thermodynamic properties can be derived. By finding the values of $a$ and $\mathcal{F}$ that allows $W$ to take its stationary value, we obtain an approximate theory that naturally and selfconsistently accounts for the effects of the reaction field in a polar fluid. In Sec. II C, we apply the theory to a polar fluid in a weak applied field and explore its various properties.

\section{The linear response regime}

\section{The solution of variational parameters}

When the applied field is weak, the polarization of the fluid responds to the applied field linearly. In this linear response regime, we may solve the constitutive relations, Eqs. (20a) and (20b), to first order in the applied field. At this order, Eq. (20a) reduces to

$$
\begin{aligned}
\frac{\beta V}{\varepsilon_{0} \tilde{a}(\mathbf{k})} & =\lambda \int d \mathbf{r} \int d \Omega \boldsymbol{\mu} \cdot \widetilde{\mathcal{T}}(\mathbf{k}) \cdot \boldsymbol{\mu} e^{-\frac{1}{2} \boldsymbol{\mu} \cdot \mathcal{T}_{R} \cdot \boldsymbol{\mu}}(1+\gamma(\mathbf{r}) \cdot \boldsymbol{\mu}) \\
& =\lambda \int d \mathbf{r} \int d \Omega \boldsymbol{\mu} \cdot \widetilde{\mathcal{T}}(\mathbf{k}) \cdot \boldsymbol{\mu} e^{-\frac{1}{2} \boldsymbol{\mu} \cdot \mathcal{T}_{R} \cdot \boldsymbol{\mu}},
\end{aligned}
$$

where the second equality results from the integral over an odd power of the permanent dipole moment. As such, the solution to $\tilde{a}(\mathbf{k})$ is isotropic with respect to $\mathbf{k}$. Consequently, the effective self-interaction tensor $\mathcal{T}_{R}$ is also isotropic, and it may be written as 


$$
\mathcal{T}_{R}=\int \frac{d \mathbf{k}}{(2 \pi)^{3}} \frac{\varepsilon_{0} \tilde{a}(\mathbf{k})}{\beta+\varepsilon_{0} \tilde{a}(\mathbf{k})} \tilde{\mathcal{T}}(\mathbf{k})=T_{R} \mathbb{1},
$$

where $T_{R}$ is a scalar characterizing the strength of the effective self-interaction.

The isotropic nature of $\mathcal{T}_{R}$ results in considerable simplifications in the solution of the constitutive relations since the term $\frac{1}{2} \boldsymbol{\mu} \cdot \mathcal{T}_{R} \cdot \boldsymbol{\mu}=\frac{1}{2} T_{R} \bar{\mu}^{2}$ is now independent of the direction of $\boldsymbol{\mu}$. The relation between the fluid density and the fugacity is, to the linear order in the applied field,

$$
\rho=4 \pi \lambda e^{-\frac{1}{2} T_{R} \bar{\mu}^{2}}
$$

The constitutive relations Eqs. (20a) and (20b) are now simplified to

$$
\frac{\beta}{\varepsilon_{0} \tilde{a}(\mathbf{k})}=y
$$

and

$$
\widetilde{\mathcal{E}}_{0}(\mathbf{k})-\widetilde{\mathcal{F}}(\mathbf{k})=(1+y) \tilde{\gamma}(\mathbf{k}),
$$

where $y=\beta \rho \bar{\mu}^{2} / 3 \varepsilon_{0}$ is a dimensionless parameter characterizing the strength of dipolar interactions in the absence of the applied field.

As seen in Eq. (25a), the solution to the variational parameter $\tilde{a}(\mathbf{k})$ is independent of $\mathbf{k}$ in the linear response regime. The solution to the other variational parameter, $\widetilde{\mathcal{F}}(\mathbf{k})$, can be obtained by substituting the expression for $\tilde{\gamma}(\mathbf{k})$ in Eq. (19) into Eq. (25b). This substitution leads to

$$
\left(1+y \frac{\varepsilon_{0}}{\beta} \widetilde{\mathcal{T}}(\mathbf{k})\right) \widetilde{\mathcal{F}}(\mathbf{k})=-y\left(1-\frac{\varepsilon_{0}}{\beta} \widetilde{\mathcal{T}}(\mathbf{k})\right) \widetilde{\mathcal{E}}_{0}(\mathbf{k})
$$

which gives the solution

$$
\widetilde{\mathcal{F}}(\mathbf{k})=\widetilde{\mathbf{K}}(\mathbf{k}) \widetilde{\mathcal{E}}_{0}(\mathbf{k}),
$$

where

$$
\widetilde{\mathbf{K}}(\mathbf{k})= \begin{cases}-y\left(\mathbb{1}-\frac{\varepsilon_{0}}{\beta} \widetilde{\mathcal{T}}(\mathbf{k})\right) & \text { for } \mathbf{k} \neq \mathbf{0}, \\ -\left(\frac{2 y}{y+3}\right) \mathbb{1} & \text { for } \mathbf{k}=\mathbf{0} .\end{cases}
$$

The details for solving Eq. (26) are given in Appendix D.

One may be intrigued that the relation between $\widetilde{\mathcal{F}}(\mathbf{k})$ and $\widetilde{\mathcal{E}}_{0}(\mathbf{k})$ takes different forms when $\mathbf{k} \neq \mathbf{0}$ and $\mathbf{k}=\mathbf{0}$. Mathematically, this difference is due to the singularity in $\widetilde{\mathcal{T}}(\mathbf{k})$ at $\mathbf{k}=\mathbf{0}$. Physically, this feature has a deep origin rooted in the existence of a monopole source for electric fields, which places a requirement on the value of $\widetilde{\mathcal{T}}(\mathbf{k})$ at $\mathbf{k}=\mathbf{0}$ so as to satisfy the electrostatic Poisson equation. Readers may refer to Ref. 61 for a more in-depth discussion on this.

\section{The polarization and the dielectric constant}

The polarization of a fluid under an electric field can be obtained by taking the derivative of the variational grand potential with respect to the applied field using the statisticalmechanical relation

$$
\mathbf{P}(\mathbf{r})=-\frac{\delta \beta W}{\delta \mathcal{E}_{0}(\mathbf{r})}
$$

In the $\mathbf{k}$-space, the above expression is equivalently written as

$$
\widetilde{\mathbf{P}}(\mathbf{k})=-(2 \pi)^{3} \frac{\partial \beta W}{\partial \widetilde{\mathcal{E}}_{0}(-\mathbf{k})} .
$$

By performing the derivative to first order in the applied field, we obtain the following expression for the polarization:

$$
\begin{aligned}
\widetilde{\mathbf{P}}(\mathbf{k})= & \left(\frac{y}{y+1}\right)^{2}\left(\frac{\varepsilon_{0}}{\beta}\right)^{2} \widetilde{\mathcal{T}}(\mathbf{k})(\mathbb{1}-\widetilde{\mathbf{K}}(\mathbf{k}))^{2} \widetilde{\mathcal{E}}_{0}(\mathbf{k}) \\
& +\frac{\varepsilon_{0}}{\beta} y\left[\mathbb{1}-\left(\frac{y}{y+1}\right) \frac{\varepsilon_{0}}{\beta} \widetilde{\mathcal{T}}(\mathbf{k})(\mathbb{1}-\widetilde{\mathbf{K}}(\mathbf{k}))\right]^{2} \widetilde{\mathcal{E}}_{0}(\mathbf{k}) .
\end{aligned}
$$

Simplification of the above expression with the use of Eq. (4) leads to

$$
\widetilde{\mathbf{P}}(\mathbf{k})=\left(\frac{\varepsilon_{0}}{\beta}\right) y \widetilde{\mathcal{E}}_{0}(\mathbf{k})-\left(\frac{\varepsilon_{0}}{\beta}\right)^{2} \frac{y^{2}}{1+y}\left(\mathbb{1}-\widetilde{\mathbf{K}}(\mathbf{k})^{2}\right) \widetilde{\mathcal{T}}(\mathbf{k}) \widetilde{\mathcal{E}}_{0}(\mathbf{k})
$$

The expressions for polarization allow us to extract the dielectric constant of the fluid. To do so, we note that the electric susceptibility matrix is defined through $\widetilde{\mathbf{P}}(\mathbf{k})=\varepsilon_{0} \widetilde{\chi}_{0} \widetilde{\mathcal{E}}_{0}(\mathbf{k}) / \beta$. From Eq. (32), we identify the electric susceptibility matrix as

$$
\widetilde{\chi}_{0}(\mathbf{k})=y \mathbb{1}-\frac{y^{2}}{1+y}\left(\mathbb{1}-\widetilde{\mathbf{K}}(\mathbf{k})^{2}\right) \frac{\varepsilon_{0}}{\beta} \widetilde{\mathcal{T}}(\mathbf{k}) .
$$

Then, the dielectric constant can be found using the relation ${ }^{39}$

$$
\frac{(\varepsilon-1)(2 \varepsilon+1)}{\varepsilon}=\operatorname{tr} \widetilde{\chi}_{0}(\mathbf{k}=\mathbf{0}) .
$$

Making use of $\widetilde{\mathcal{T}}(\mathbf{k}=\mathbf{0})=\int d \mathbf{r} \mathcal{T}(\mathbf{r})=\left(\beta / 3 \varepsilon_{0}\right) \mathbb{1}$, we obtain the following simple expression for the dielectric constant:

$$
\frac{(\varepsilon-1)(2 \varepsilon+1)}{\varepsilon}=3 y\left(\frac{2 y^{2}+3 y+9}{(y+3)^{2}}\right) \text {. }
$$

This is to be compared with the well-known Onsager result

$$
\frac{(\varepsilon-1)(2 \varepsilon+1)}{\varepsilon}=3 y \text {. }
$$

Equation (35) for the dielectric constant is a key result of our theory. It predicts the dielectric constant of a polar liquid based on the magnitude of the permanent dipole moment $\bar{\mu}$ of the constituting molecule, the density of the fluid $\rho$, and the temperature $T$. When the system is sufficiently dilute, the expression reduces to $\varepsilon=1+y$ to the first order in $y$, recovering the Debye theory for a dipolar gas. In Sec. III, we will apply Eq. (35) to calculate the dielectric constants for a range of nonpolarizable liquid models, examine their agreement with the values obtained from simulation, and compare the prediction of Eq. (35) to the results given by the Onsager equation and previous field-theoretic approaches.

Note that one recovers Debye's result for the dielectric constant $\varepsilon=1+y$ if we take $\widetilde{\mathbf{K}}(\mathbf{k})=\mathbf{0}$. This is to be expected since setting $\widetilde{\mathbf{K}}(\mathbf{k})=\mathbf{0}$ amounts to ignoring the reaction field. It is interesting that Onsager's result corresponds to $\widetilde{\mathbf{K}}(\mathbf{k})=-\mathbb{1}$. The reason for this is subtle and has to do with the equivalence between the applied field in our system and the cavity field in Onsager's construct, which we will elaborate further in the following paragraph. 
The equivalence between the applied field in our system and the cavity field in Onsager's construct can be seen by considering a macroscopically sized spherical system in an applied field $\mathbf{E}_{0}$ (and the radius of the system can be taken to infinity eventually). A uniform polarization develops in the liquid because of the applied field. If we carve out a molecular-sized cavity at the center of the liquid, as constructed by Onsager, the field $\mathbf{E}_{c}$ inside the cavity equals $\mathbf{E}_{0}$ because a spherical shell of uniform polarization does not exert a field at the center. ${ }^{62}$ Because of this equivalence between the applied and the cavity field, when $\widetilde{\mathbf{K}}(\mathbf{k})=-\mathbb{1}$, the polarization is $\mathbf{P}=\varepsilon_{0} y \mathbf{E}_{0}$ $=\varepsilon_{0} y \mathbf{E}_{c}$ from Eq. (32). This means that, when we set $\widetilde{\mathbf{K}}(\mathbf{k})=-\mathbb{1}$ in our theory, the directing field-which exerts a torque on the dipoles-is equal to the cavity field $\mathbf{E}_{c}$. Since in Onsager's theory, the cavity field is also the directing field (for a nonpolarizable liquid), setting $\widetilde{\mathbf{K}}(\mathbf{k})=-\mathbb{1}$ in our theory recovers Onsager's result.

To further explore the connection between our theory and classical linear dielectric theory, we consider the case of a uniform applied field. Using Eq. (32) for $\mathbf{k}=\mathbf{0}$ and recalling $\mathcal{E}_{0}=\beta \mathbf{E}_{0}$, the polarization is

$$
\mathbf{P}=\varepsilon_{0} \frac{y\left(2 y^{2}+3 y+9\right)}{(y+3)^{2}} \mathbf{E}_{0} .
$$

By the connection between the cavity field and the electric (Maxwell) field $\mathbf{E}$ in Onsager's treatment, ${ }^{63}$ we have

$$
\mathbf{E}=\frac{(2 \varepsilon+1)}{3 \varepsilon} \mathbf{E}_{0} .
$$

On the other hand, for a linear dielectric medium, the polarization is related to the electric field in the material by

$$
\mathbf{P}=\varepsilon_{0}(\varepsilon-1) \mathbf{E} .
$$

Combining the above three equations, we obtain our result for the dielectric constant, Eq. (35).

\section{The reaction field}

The concept of the reaction field, first introduced by Onsager, ${ }^{16}$ was a major advancement in our understanding of dielectric fluids. The concept is most easily illustrated by considering a tagged dipole in the fluid. The orientation of this dipole influences the orientation of other dipoles in its surrounding, which then exerts a reaction field back on the tagged dipole. This reaction field thus always points in the same direction as the tagged dipole, and it can be formally written as

$$
\mathcal{R}=\beta v \mu,
$$

where we have defined the reaction field factor $v$.

We can determine the reaction field based on the effective single-particle potential in Eq. (17), which gives the free energy of a dipole in the absence of an applied field as

$$
\begin{aligned}
f_{0} & =\frac{1}{2} \boldsymbol{\mu} \cdot \mathcal{T}_{R} \cdot \boldsymbol{\mu} \\
& \left.=\frac{1}{2} \boldsymbol{\mu} \cdot \mathcal{T}(\mathbf{0}) \cdot \boldsymbol{\mu}-\frac{1}{2} \boldsymbol{\mu} \cdot\left(\int \frac{d \mathbf{k}}{(2 \pi)^{3}} \frac{\beta}{\beta+\varepsilon_{0} \tilde{a}(\mathbf{k})} \widetilde{\mathcal{T}} \mathbf{k}\right)\right) \cdot \boldsymbol{\mu} .
\end{aligned}
$$

The first term $\frac{1}{2} \boldsymbol{\mu} \cdot \boldsymbol{\mathcal { T } ( \mathbf { 0 } )} \cdot \boldsymbol{\mu}$ represents the self-energy of the dipole, while the second term is due to the reaction field. Since the free energy for the interaction between the dipole and the reaction field is $-\frac{1}{2} \boldsymbol{\mu} \cdot \mathcal{R}=-\frac{1}{2} \beta \nu \boldsymbol{\mu}^{2},{ }^{17}$ we identify the reaction field factor to be

$$
v=\int \frac{d \mathbf{k}}{(2 \pi)^{3}} \frac{1}{\beta+\varepsilon_{0} \tilde{a}(\mathbf{k})} \tilde{\mathcal{T}}(\mathbf{k})=\frac{1}{3 \varepsilon_{0} b^{3}} \frac{y}{y+1},
$$

where we have introduced a microscopic cutoff $b$ through $\int 1 d \mathbf{k} /(2 \pi)^{3}=1 / b^{3}$, which defines the length scale of the selfinteraction. Physically we expect $b$ to be on the order of the molecular size; for a liquid, we may set $b^{3}=1 / \rho$.

In Onsager's treatment, the reaction field was computed by placing a tagged dipole inside a spherical vacuum cavity of size $b^{3}$ in a medium of dielectric constant $\varepsilon$. Using simple electrostatics, the reaction field factor can be shown to be $v=\frac{1}{3 \varepsilon_{0} b^{3}} \frac{2(\varepsilon-1)}{2 \varepsilon+1}$. With our field-theoretic approach, the artificial construct of a vacuum cavity is avoided. The resulting reaction field agrees with Onsager's in the limit of strong dielectric $(y \gtrsim 3)$, but is weaker than Onsager's for weak dielectrics $(y \lesssim 3)$.

\section{The free energy}

It is of interest to consider the free energy in the linear response regime. The general expression can be obtained from the grand potential in Eq. (16) combined with the variational conditions Eqs. (20a) and (20b). Here we consider the simple case of a uniform applied field $\mathbf{E}_{0}$. The total grand potential to quadratic order is

$\beta W=-\frac{1}{2} \frac{V}{b^{3}}\left[\ln \left(\frac{1}{y+1}\right)+\frac{y}{y+1}\right]-\frac{1}{2} \beta V\left(\frac{3 y}{y+3}\right) \varepsilon_{0} E_{0}^{2}-\rho V$,

where $E_{0}=\left|\mathbf{E}_{0}\right|$ is the magnitude of the applied field and $T_{R}$ is evaluated based on Eq. (23), which gives

$$
T_{R}=\frac{\beta}{3 \varepsilon_{0} b^{3}(1+y)} .
$$

Performing the Legendre transform to the same order, we obtain the final Helmholtz free energy

$$
\begin{aligned}
\beta F & =\beta W+\beta \mu N \\
& =\frac{V}{2 b^{3}} \ln (1+y)-\frac{1}{2} \beta V\left(\frac{3 y}{y+3}\right) \varepsilon_{0} E_{0}^{2}+N \ln \left(\frac{N \Lambda^{3}}{V}\right)-N .
\end{aligned}
$$

Thus, the Helmholtz free energy due to the applied field is

$$
\beta F_{E_{0}}=-\frac{1}{2} \beta V\left(\frac{3 y}{y+3}\right) \varepsilon_{0} E_{0}^{2}
$$

which suggests that the free energy decreases when an electric field is applied. This is to be expected because the applied field induces a polarization in a direction that is energetically favorable.

We have thus developed the theoretical framework in this section. The framework includes a master variational grand potential for a general polar liquid, and from this grand 
potential, all other thermodynamic quantities can be derived. By considering a weak applied field, we have derived an expression for the dielectric constant and this is a key result of the theory. In Sec. III, we apply our expression for the dielectric constant to a range of nonpolarizable liquid models and examine their agreement with the simulation-obtained values.

\section{DIELECTRIC CONSTANTS OF MODEL LIQUIDS}

A key result of our theory is the expression for the dielectric constant of a polar fluid presented in Eq. (35). This expression computes the dielectric constant based on the number density and the permanent dipole moment of the fluid molecules, as well as the temperature of the liquid. In this section, we apply the expression to calculate the dielectric constants of model liquids of small organic molecules and compare our results to the predictions of the Onsager equation and previous field-theoretic approaches. Because all real molecules are polarizable but our current theory only considers permanent dipoles, it is more appropriate to use nonpolarizable liquid models as the basis for comparison. Among the major forcefields available today, two of the commonly used forcefields for small molecules are the OPLS/AA (all-atom optimized potential for liquid simulations) and the GAFF (generalized Amber force field) models. The dielectric constants for over a hundred molecules with these two forcefields have been computed by Caleman et al. ${ }^{53}$ using computer simulation. To the best of our knowledge, Ref. 53 provides the most extensive set of dielectric constants obtained from computer simulation; therefore, we compare our predicted dielectric constants with the corresponding values reported in Ref. 53.

As the dielectric constants of the model liquids vary over two orders of magnitude, we plot the results on a logarithmic scale. All available simulation data reported in Ref. 53 are included for comparison. In Fig. 1(a), the dielectric

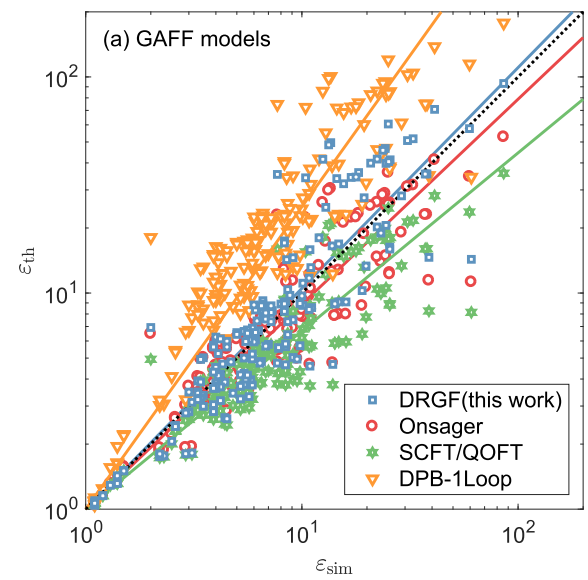

constants of the GAFF liquid models calculated using our theory [Eq. (35)] are plotted against the corresponding simulation data as blue squares scattered on a log-log scale. Details of the parameters used in the calculation, and the numerical values of the dielectric constants from both our theoretical prediction and from the computer simulation, are provided in the supplementary material. A best-fit line is drawn through the scattered points, and the line has a slope of 1.02. In view of the highly coarse-grained nature of our theory, the agreement between the theory and the simulation is quite good. The actual molecules in the simulation include detailed molecular structures; some structures enhance dipole-dipole correlations (as in the case of some hydrogen bonding liquids), while others hinder dipole-dipole interactions. Such molecular details are not captured in our coarse-grained theory. Therefore, it is not surprising that the theory results in an underestimate of the dielectric constants for some liquids and an overestimate for others. In Fig. 1(b), we similarly plot the theoretical vs. simulation dielectric constants for the OPLS/AA liquid models. Details of the parameters involved and the results for individual molecules are also provided in the supplementary material. For the OPLS/AA liquid models, the line of best-fit through the theory vs. simulation values has a slope of 0.98 , which again demonstrates the overall good predictive power of our theory.

As mentioned in the Introduction, there have been a number of attempts in modeling the dielectric response in softmatter systems. The simplest approach is the self-consistentfield theory (SCFT), which treats the field-based partition function using the saddle-point approximation. Examples of this approach include the ionic screening theory by Coalson and Duncan, ${ }^{43,44}$ the dipolar Poisson-Boltzmann equation by Abrashkin et al. ${ }^{45}$ the dipolar self-consistent-field theory by Nakamura et al. ${ }^{46}$ and the generalized theory for polymers containing dipoles on the backbone by Kumar et al. ${ }^{47}$ The SCFT gives the following simple expression for the dielectric constant:

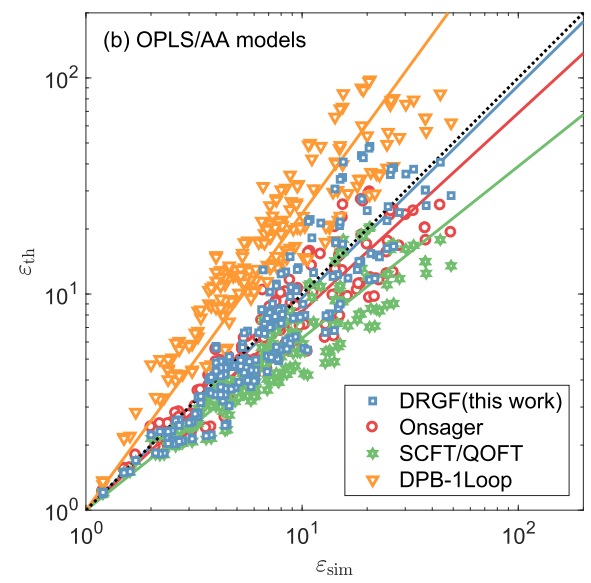

FIG. 1. Dielectric constants of liquid models calculated using the theories $\varepsilon_{\text {th }}$ vs. values obtained from simulations $\varepsilon_{\text {sim }}$ plotted on a log-log scale. The liquid models are (a) the GAFF (generalized Amber force field) models and (b) the OPLS/AA (all-atom optimized potential for liquid simulations) models. The theories used included our dipolar renormalized gaussian fluctuation theory (DRGF, blue open squares), the Onsager equation (red open circles), the self-consistent field theory or the quadratic order field theory (SCFT/QOFT, green stars), and the Dipolar Poisson Boltzmann approach expanded to one-loop level (DPB-1Loop, orange open inverted triangles). Respectively, the lines of best fit for $\log \varepsilon_{\text {th }}$ vs. $\log \varepsilon_{\text {sim }}$ for the DRGF theory, the Onsager equation, the SCFT/QOFT theory, and the DPB-1Loop approach have slopes 1.02, 0.95, 0.82, and 1.40 for the GAFF models in (a) and 0.98, 0.92, 0.80, and 1.37 for the OPLS/AA models in (b). The black dashed line has a slope of 1 on the log-log scale, plotted for reference purposes. 


$$
\varepsilon=1+\frac{\beta \bar{\mu}^{2} \rho}{3 \varepsilon_{0}} .
$$

Since SCFT is a mean-field theory that ignores fluctuations, it is not surprising that the reaction field is not accounted for in SCFT. Indeed, the SCFT result is the same as the Debye theory, which is applicable only when the molecules are sufficiently dilute. In a dense liquid, the presence of the reaction field enhances dipole-dipole correlations; therefore, the SCFT is expected to underestimate the liquid dielectric constants. In Figs. 1(a) and 1(b), we plot the SCFT dielectric constants against the simulation values for the GAFF and the OPLS/AA models, respectively. The best-fit lines through the data points are found to have slopes 0.82 and 0.80 , respectively, confirming our expectation that the SCFT indeed underestimates the dielectric constants.

Very recently, Martin et al. presented a dielectric theory for polarizable soft matter. In their work, a field-theoretic approach is used to describe a system of polarizable "beads" and a simple gaussian approximation is employed to treat the field-based partition function. Since the field-theoretic action is kept to the quadratic order in the work, we will call the theory a quadratic-order field theory (QOFT). The work focuses primarily on fluids of induced dipoles, but the authors have also provided the partition function for a system with only permanent dipoles. By comparing Eqs. (10) and (14) in Ref. 50 to second order in the field $\bar{\phi}$ in the exponent, we find that polarizability $\alpha$ in the induced-dipole-only case is algebraically equivalent to $\bar{\mu}^{2} / 3$ in the permanent-dipole-only case. ${ }^{64}$ Therefore, the dielectric constant for the permanent-dipole-only case given by the QOFT theory is [based on Eq. (44) in Ref. 50, converted to SI unit]

$$
\varepsilon=1+\frac{\beta \bar{\mu}^{2} \rho}{3 \varepsilon_{0}}
$$

which is, surprisingly, the same as the SCFT result. We are unable to comment on the exact reason for the gaussian approximation to reproduce the SCFT result, but we note that the electric potential field fluctuates around zero in the gaussian approximation and this may be insufficient for interactions beyond the saddle-point approximation to be taken into account. As the QOFT result is the same as the SCFT results, the comparison in Fig. 1 for the SCFT applies equally to the QOFT results.

Another field-theoretic dielectric theory developed in recent years is the Dipolar Poisson-Boltzmann (DPB) approach by Levy, Andelman, and Orland. ${ }^{48,49}$ In their work, a system of ions and dipoles are considered and field-theoretic methods are applied to derive the field-based grand partition function. The authors expand the free energy to first order in a loop expansion to obtain a closed form formula for the dielectric constant. For a system of only permanent dipole moments, the dielectric constant is given by

$$
\varepsilon=1+\varepsilon_{1}+\frac{\varepsilon_{1}^{2}}{1+\varepsilon_{1}} \frac{4 \pi}{3 \rho b^{3}},
$$

where $\varepsilon_{1}=\beta \rho \bar{\mu}^{2} / 3 \varepsilon_{0}$ is the self-consistent-field contribution to the dielectric constant and $b$ is a cut-off length in the momentum integration which is considered to be related to the size of the molecule. The cut-off length $b$ is a fitting parameter in the DPB approach, and we have found that the value of $\varepsilon$ is very sensitive to the value of $b$ used. To apply the theory free of any adjustable parameters, we let $b^{3}=1 / \rho$ so that $b^{3}$ describes the average size of each molecule. The dielectric constant calculated using Eq. (49) is referred to as DPB-1Loop in the plots. In Figs. 1(a) and 1(b), we plot the DPB-1Loop dielectric constants against the simulation values for the GAFF and the OPLS/AA models, respectively. The best-fit lines through the DPB-1Loop data points are found to have slopes 1.40 and 1.37 for the GAFF and the OPLS/AA models, respectively, showing that the DPB-1Loop approach significantly overestimates the dielectric constants of liquid models.

In classical statistical-mechanical treatments of dielectric liquids, a major improvement over Debye's dilute-limit theory was provided by Onsager. As the Onsager theory involves the same number of parameters as in our theory and is the best known analytical theory for dielectric constants, we compare the predictions from our theory with those from the Onsager equation. In Figs. 1(a) and 1(b), we plot the dielectric constants predicted by the Onsager equation vs. the simulation values, where the lines of best-fit have slopes 0.95 and 0.92 for the GAFF and the OPLS/AA models, respectively. These are to be compared to the slopes of 1.02 and 0.98 by our theory. The level of agreement between theory and simulation is comparable between the Onsager equation and our theory, but our theory produces slightly better agreement. More importantly, by treating all particles equivalently, our theory provides a greater degree of self-consistency than Onsager's approach, which requires the artificial construct of a cavity around a tagged molecule. One clear advantage of our theory is the ease with which to generalize to liquid mixtures.

On closer examination of the variational approach that we have developed in this work, we note that the inclusion of the variational parameter $\mathcal{F}$ is responsible for the improvement over previous field-theoretic approaches. If we set the field $\mathcal{F}$ to zero [i.e., setting $\widetilde{\mathbf{K}}(\mathbf{k})=\mathbf{0}$ in Eq. (28)], we recover the SCFT result for the dielectric constant in Eq. (47). This suggests that in constructing the variational reference action $L_{0}$ in Eq. (12), it is crucial to allow the field $i \mathcal{G}$ to fluctuate around a nonzero average value $\mathcal{F}$ so that the effects of reaction field can be accounted for.

The effect of $\mathcal{F}$ may be further understood by looking into the single-particle partition function. In the exact fieldtheoretic action [Eq. (11)], the last term is the single-particle partition function under the auxiliary field $i \mathcal{G}$

$$
q_{\text {exact }}=\lambda \int d \mathbf{r} \int d \Omega e^{i \boldsymbol{\mu} \cdot \mathcal{G}(\mathbf{r})},
$$

whereas in the reference action that we proposed, the last term is in the form of the single-particle partition function under the field $i \mathcal{G}-\mathcal{F}$ expanded to second order in the fields since

$$
\begin{aligned}
q_{\mathrm{ref}} & =\lambda \int d \mathbf{r} \int d \Omega e^{\boldsymbol{\mu} \cdot[i \mathcal{G}(\mathbf{r})-\mathcal{F}(\mathbf{r})]} \\
& =\text { constant }+\frac{1}{2} \frac{4 \pi \lambda \bar{\mu}^{2}}{3} \int d \mathbf{r}[i \mathcal{G}(\mathbf{r})-\mathcal{F}(\mathbf{r})]^{2}+\cdots
\end{aligned}
$$


Here, the linear term vanishes due to the integral over the solid angle of the dipole. Apparently, in the reference action, the dipole is now in a "corrected" fluctuating field $i \mathcal{G}-\mathcal{F}$. This "corrected" field is in a similar spirit as Onsager's approach, which established that only a portion of the local field acting on a dipole exerts a torque on the dipole (the reaction field is always in the same direction as the dipole and, therefore, it does not exert any torque). However, our theory is very different from Onsager's in the approach for incorporating reactionfield effects-while Onsager carefully examined the forces on a tagged molecule inside an artificially constructed vacuum cavity, in our theory, the reaction field arises naturally from properly treating the fluctuation-correlation in the entire fluid system.

\section{CONCLUSIONS}

In this work, we have developed a coarse-grained theory for pure polar liquids. By introducing a gaussian reference action in the field-theoretic representation, we account for the electrostatic interactions through a self-consistent variational procedure. Such a procedure allows us to treat all particles on the same footing and thus it is free of the artificial construct of a cavity, differing from Onsager's dielectric theory which singles out a particle in the liquid. Through the field-interaction tensor and the average fluctuating field as variational parameters, our variational procedure mathematically renormalizes the dipole-dipole interaction strength and the local field acting on each dipole and, as a result, the procedure naturally accounts for the reaction field arising from the dipole-dipole interactions.

A key result in our work is the expression for dielectric constant of a pure liquid, Eq. (35). This is a simple analytical expression that predicts the dielectric constant with only two readily available parameters - the permanent dipole moment and the density of the moleculesrequiring no adjustable parameters. For an extensive set of nonpolarizable model liquids, we have computed the dielectric constants with our theory, and the best-fit line through the theoretically predicted dielectric constants vs. the corresponding simulation-observed values has a slope very close to 1 on a log-log plot. We have also compared the predictions of our theory to earlier dielectric theories derived using other field-theoretic approximations and the results have shown that our theory produces better agreement with the simulation results.

We have applied the variational theory to homogeneous pure polar fluids in a weak applied field, where the polarization responds linearly to the applied field. Beyond this, the full grand potential Eq. (16), along with the two constitutive relations, Eqs. (20a) and (20b), is applicable to systems in the presence of strong and/or nonuniform applied fields. Therefore, the theory can be readily applied, for example, to study dipolar fluid in the vicinity of strongly charged ions.

Since the derivation of our theory does not require singling out a special tagged molecule or the use of a cavity construct as in the theory by Onsager, it is straightforward to extend the DRGF theory to liquid mixtures. Such an extension is important because the parameter space for mixtures-the different molecular species and different compositions-is too enormous to be representatively covered by computer simulation; a simple theory is highly useful and desirable. In addition, our theory can be extended to polarizable liquids, where the polarizability and the van der Waals interactions resulting from it have proved to be important. ${ }^{65}$ Very recently, Grzetic, Delaney, and Fredrickson applied the QOFT framework to explore the connection between polarizability and the effective $\chi$ parameter in non-polar polarizable systems, ${ }^{66}$ our current work can be extended to polarizable systems and applied in a similar pursuit. We reserve these efforts for future work.

The past two decades have seen great success in the application of field theoretical methods to the study of a vast range of problems in the areas of soft matter, including polymers, liquid crystals, and ionic solutions. ${ }^{40,42,67}$ While earlier work focused on the self-consistent mean-field approximations, ${ }^{45,68-70}$ considerable progress has been made in understanding the nontrivial and sometimes qualitative effects due to fluctuations..$^{51,71,72}$ The advent of field theoretical simulations ${ }^{41}$ has further expanded the capabilities of the fieldtheoretical methods and allowed some hitherto impossible phenomena to be described..$^{73-76}$ In this work, we have shown that field-theoretical techniques can naturally capture the nontrivial reaction field-another manifestation of fluctuation effects-in the dielectric response of molecular liquids. In combination with field-theoretic representation of other degrees of freedom, the present theory contributes to the general field-theoretical coarse-grained modeling of soft-matter systems to allow the description of the dielectric response of the materials.

\section{SUPPLEMENTARY MATERIAL}

The supplementary material contains the data and the parameters used in Fig. 1.

\section{ACKNOWLEDGMENTS}

We thank Issei Nakamura, Rui Wang, Kevin Shen, Jian Jiang, Nayef Alsaifi, and Pengfei Zhang for helpful discussions. B.Z. gratefully acknowledges the support by an A-STAR fellowship. Acknowledgement is also made to the donors of the American Chemical Society Petroleum Research Fund for partial support of this research.

\section{APPENDIX A: IDENTITY TRANSFORMATION OF THE GRAND PARTITION FUNCTION}

Since the dipole-dipole interaction tensor $\mathbf{T}(\mathbf{r})$ does not have an inverse operator, we perform the identity transformations using the Faddeev-Popov method ${ }^{40,42}$ by introducing the $\delta$-functional into the partition function in Eq. (10),

$$
\mathbf{1}=\int \mathcal{D} \mathbf{P} \delta[\mathbf{P}(\mathbf{r})-\hat{\mathbf{P}}(\mathbf{r})]=\int \mathcal{D} \mathbf{P} \int \mathcal{D} \mathcal{G} e^{i \int d \mathbf{r} \mathcal{G}(\mathbf{r}) \cdot[\mathbf{P}(\mathbf{r})-\hat{\mathbf{P}}(\mathbf{r})]},
$$


where $\delta[\mathbf{f}(\mathbf{r})]$ is the generalization of the multivariate $\delta$ function. With the $\delta$-functional in Eq. (A1), we transform the Boltzmann factor as

$$
\begin{aligned}
e^{-\beta U}= & \int \mathcal{D} \mathbf{P} \int \mathcal{D} \mathcal{G} e^{-\frac{1}{2} \int d \mathbf{r} \int d \mathbf{r}^{\prime} \mathbf{P}(\mathbf{r}) \mathcal{T}\left(\mathbf{r}-\mathbf{r}^{\prime}\right) \mathbf{P}\left(\mathbf{r}^{\prime}\right)} \\
& \times e^{-i \int d \mathbf{r} \mathbf{P}(\mathbf{r}) \cdot \mathcal{G}(\mathbf{r})+\int d \mathbf{r} \mathbf{P}(\mathbf{r}) \cdot \mathcal{E}_{0}(\mathbf{r})+i \int d \mathbf{r} \hat{\mathbf{P}}(\mathbf{r}) \cdot \mathcal{G}(\mathbf{r})},
\end{aligned}
$$

where only the last term in the exponential depends explicitly on the instantaneous molecular configuration. Use of Eq. (A2) in the Boltzmann factor in $Z(N)$ in Eq. (6) leads to

$$
\begin{aligned}
Z(N)= & \int \mathcal{D} \mathbf{P} \int \mathcal{D} \mathcal{G} e^{-\frac{1}{2} \int d \mathbf{r} \int d \mathbf{r}^{\prime} \mathbf{P}(\mathbf{r}) \mathcal{T}\left(\mathbf{r}-\mathbf{r}^{\prime}\right) \mathbf{P}\left(\mathbf{r}^{\prime}\right)} \\
& \times e^{-i \int d \mathbf{r} \mathbf{P}(\mathbf{r}) \cdot \mathcal{G}(\mathbf{r})+\int d \mathbf{r} \mathbf{P}(\mathbf{r}) \cdot \mathcal{E}_{0}(\mathbf{r})} \\
& \times\left\{\frac{1}{4 \pi \Lambda^{3}} \int d \mathbf{r} \int d \Omega e^{i \boldsymbol{\mu} \cdot \mathcal{G}(\mathbf{r})}\right\}^{N} .
\end{aligned}
$$

Substituting Eq. (A3) into Eq. (5) and summing over all $N$ leads to the field-based grand partition function and the fieldtheoretic action given by Eqs. (10) and (11).

\section{APPENDIX B: EVALUATION OF THE VARIATIONAL BOUND}

In this section, we present the evaluation of the variational grand potential $W$. The evaluation involves the operator $\mathbf{Q}=\mathcal{T}+\mathcal{A}$ and its inverse $\mathbf{Q}^{-1}$. We start by deriving an expression for $\mathbf{Q}^{-1}$ in the Fourier space

$$
\begin{aligned}
\widetilde{\mathbf{Q}}^{-1}(\mathbf{k}) & =[\widetilde{\mathcal{T}}(\mathbf{k})+\widetilde{\mathcal{A}}(\mathbf{k})]^{-1}=[\widetilde{\mathcal{T}}(\mathbf{k})+\tilde{a}(\mathbf{k}) \mathbb{1}]^{-1}=\frac{1}{\tilde{a}(\mathbf{k})}\left[\mathbb{1}+\tilde{a}(\mathbf{k})^{-1} \widetilde{\mathcal{T}}(\mathbf{k})\right]^{-1} \\
& =\frac{1}{\tilde{a}(\mathbf{k})}\left[\mathbb{1}-\tilde{a}(\mathbf{k})^{-1} \widetilde{\mathcal{T}}(\mathbf{k})+\tilde{a}(\mathbf{k})^{-2} \widetilde{\mathcal{T}}(\mathbf{k})^{2}-\tilde{a}(\mathbf{k})^{-3} \widetilde{\mathcal{T}}(\mathbf{k})^{3}+\cdots\right] \\
& =\frac{1}{\tilde{a}(\mathbf{k})}\left[\mathbb{1}-\left(\frac{\beta}{\varepsilon_{0} \tilde{a}(\mathbf{k})}\right) \frac{\mathbf{k} \mathbf{k}}{k^{2}}+\left(\frac{\beta}{\varepsilon_{0} \tilde{a}(\mathbf{k})}\right)^{2} \frac{\mathbf{k} \mathbf{k}}{k^{2}}-\left(\frac{\beta}{\varepsilon_{0} \tilde{a}(\mathbf{k})}\right)^{3} \frac{\mathbf{k k}}{k^{2}}+\cdots\right] \\
& \left.=\frac{1}{\tilde{a}(\mathbf{k})}\left[\mathbb{1}-\frac{\beta}{\varepsilon_{0} \tilde{a}(\mathbf{k})} \frac{1}{1+\frac{\beta}{\varepsilon_{0} \tilde{a}(\mathbf{k})}} \frac{\mathbf{k k}}{k^{2}}\right]=\frac{1}{\tilde{a}(\mathbf{k})}\left[\mathbb{1}-\frac{\varepsilon_{0}}{\beta+\varepsilon_{0} \tilde{a}(\mathbf{k})} \widetilde{\mathcal{T}} \mathbf{k}\right)\right] .
\end{aligned}
$$

In the fourth equality above, we have used the fact that $\mathbf{k k} / k^{2}$ is a projection operator and, thus, $\left(\mathbf{k} \mathbf{k} / k^{2}\right)^{n}=\mathbf{k} \mathbf{k} / k^{2}$ for any positive integer $n$.

Since the reference action $L_{0}$ is gaussian with respect to both $\mathbf{P}$ and $\mathcal{G}$, we can evaluate $\Xi_{0}$ using standard techniques for gaussian integrals. The result is

$\Xi_{0}=\left(\frac{\operatorname{det} \mathcal{A}}{\operatorname{det}(\mathcal{T}+\mathcal{A})}\right)^{\frac{1}{2}} e^{\int d \mathbf{r} \int d \mathbf{r}^{\prime} \frac{1}{2}\left[\mathcal{E}_{0}(\mathbf{r})-\mathcal{F}(\mathbf{r})\right] \mathbf{Q}^{-1}\left(\mathbf{r}-\mathbf{r}^{\prime}\right)\left[\mathcal{E}_{0}\left(\mathbf{r}^{\prime}\right)-\mathcal{F}\left(\mathbf{r}^{\prime}\right)\right]}$,

where the determinant of a tensor $\mathbf{M}$ is given in terms of a functional integral as

$$
\begin{aligned}
(\operatorname{det} \mathbf{M})^{-\frac{1}{2}} & =\int \mathcal{D} \boldsymbol{\xi} e^{-\frac{1}{2} \int d \mathbf{r} \int d \mathbf{r}^{\prime} \boldsymbol{\xi}(\mathbf{r}) \mathbf{M}\left(\mathbf{r}-\mathbf{r}^{\prime}\right) \boldsymbol{\xi}\left(\mathbf{r}^{\prime}\right)} \\
& =\int \mathcal{D} \boldsymbol{\xi} e^{-\frac{1}{2} \int \frac{d \mathbf{k}}{(2 \pi)^{3}} \widetilde{\xi}(\mathbf{k}) \widetilde{\mathbf{M}}(\mathbf{k}) \widetilde{\boldsymbol{\xi}}(-\mathbf{k})}
\end{aligned}
$$

The factor $\operatorname{det} \mathcal{A} / \operatorname{det}(\mathcal{T}+\mathcal{A})$ can be evaluated using the "charging" method described in Appendix B of Ref. 77. Let us define $\boldsymbol{\Gamma}(\theta)=\theta \mathcal{T}+\mathcal{A}$. Then,

$$
\begin{aligned}
\ln \left(\frac{\operatorname{det} \mathcal{A}}{\operatorname{det}(\mathcal{T}+\mathcal{A})}\right) & =\ln \operatorname{det} \boldsymbol{\Gamma}(\theta=1)-\ln \operatorname{det} \boldsymbol{\Gamma}(\theta=0) \\
& =\int d \mathbf{k} \int_{\boldsymbol{\Gamma}(\theta=0)}^{\boldsymbol{\Gamma}(\theta=1)} \frac{\delta \ln \operatorname{det} \boldsymbol{\Gamma}(\theta)}{\delta \widetilde{\boldsymbol{\Gamma}}^{-1}(\mathbf{k} ; \theta)}: \delta \widetilde{\boldsymbol{\Gamma}}^{-1}(\mathbf{k} ; \theta),
\end{aligned}
$$

where the last equality is due to the chain rule and the : symbol indicates scalar product of two tensors. Using the fact that det $\boldsymbol{\Gamma}(\theta)$ can be written as a gaussian functional integral as in Eq. (B3), we have

$$
\begin{aligned}
& \frac{\delta \ln \operatorname{det} \boldsymbol{\Gamma}(\theta)}{\delta \widetilde{\boldsymbol{\Gamma}}^{-1}(\mathbf{k} ; \theta)} \\
& =\frac{\frac{1}{(2 \pi)^{3}} \int \mathcal{D} \boldsymbol{\xi} \widetilde{\boldsymbol{\xi}}(\mathbf{k}) \widetilde{\boldsymbol{\xi}}(-\mathbf{k}) e^{-\frac{1}{2} \int \frac{d \mathbf{k}^{\prime}}{(2 \pi)^{3}} \widetilde{\boldsymbol{\xi}}\left(\mathbf{k}^{\prime}\right) \widetilde{\boldsymbol{\Gamma}}^{-1}\left(\mathbf{k}^{\prime} ; \theta\right) \widetilde{\boldsymbol{\xi}}\left(-\mathbf{k}^{\prime}\right)}}{\int \mathcal{D} \boldsymbol{\xi} e^{-\frac{1}{2} \int \frac{d \mathbf{k}^{\prime}}{(2 \pi)^{3}} \widetilde{\xi}\left(\mathbf{k}^{\prime}\right) \widetilde{\boldsymbol{\Gamma}}^{-1}\left(\mathbf{k}^{\prime} ; \theta\right) \widetilde{\boldsymbol{\xi}}\left(-\mathbf{k}^{\prime}\right)}} \\
& =-\widetilde{\boldsymbol{\Gamma}}(\mathbf{k}, \theta) \delta(\mathbf{k}=0)=-\widetilde{\boldsymbol{\Gamma}}(\mathbf{k}, \theta) \frac{V}{(2 \pi)^{3}},
\end{aligned}
$$

where, in the last equality, we have used the following interpretation of $\delta(\mathbf{k}=0):^{.78}$

$$
\delta(\mathbf{k}=0)=\left.\frac{1}{(2 \pi)^{3}} \int d \mathbf{r} e^{i \mathbf{k} \cdot \mathbf{r}}\right|_{\mathbf{k}=0}=\frac{1}{(2 \pi)^{3}} \int d \mathbf{r} 1=\frac{V}{(2 \pi)^{3}} .
$$

Furthermore, an expression for $\boldsymbol{\Gamma}^{-1}$ can be derived using a similar method that is used in Eq. (B1),

$$
\widetilde{\boldsymbol{\Gamma}}^{-1}(\mathbf{k} ; \theta)=\frac{1}{\tilde{a}(\mathbf{k})}\left[\mathbb{1}-\frac{\varepsilon_{0} \theta}{\beta \theta+\varepsilon_{0} \tilde{a}(\mathbf{k})} \widetilde{\mathcal{T}}(\mathbf{k})\right] .
$$

Then, substituting Eqs. (B5) and (B7) into (B4), we have 


$$
\begin{aligned}
\ln \left(\frac{\operatorname{det} \mathcal{A}}{\operatorname{det}(\mathcal{T}+\mathcal{A})}\right) & =\int \frac{d \mathbf{k}}{(2 \pi)^{3}} \int_{\theta=0}^{1} \widetilde{\boldsymbol{\Gamma}}(\mathbf{k} ; \theta): \frac{d \widetilde{\boldsymbol{\Gamma}}^{-1}(\mathbf{k} ; \theta)}{d \theta} d \theta \\
& =\int \frac{d \mathbf{k}}{(2 \pi)^{3}} \int_{\theta=0}^{1}[\theta \widetilde{\mathcal{T}}(\mathbf{k})+\widetilde{A}(\mathbf{k})]: \frac{\varepsilon_{0}^{2}}{\left(\theta \beta+\varepsilon_{0} \tilde{a}(\mathbf{k})\right)^{2}} \widetilde{\mathcal{T}}(\mathbf{k}) d \theta \\
& =V \int \frac{d \mathbf{k}}{(2 \pi)^{3}} \frac{\varepsilon_{0}}{\beta+\varepsilon_{0} \tilde{a}(\mathbf{k})} \operatorname{tr} \widetilde{\mathcal{T}}(\mathbf{k})-V \int \frac{d \mathbf{k}}{(2 \pi)^{3}}\left[\ln \left(\frac{\varepsilon_{0} \tilde{a}(\mathbf{k})}{\beta+\varepsilon_{0} \tilde{a}(\mathbf{k})}\right)+\frac{\beta}{\beta+\varepsilon_{0} \tilde{a}(\mathbf{k})}\right]
\end{aligned}
$$

Next, we evaluate $\left\langle L-L_{0}\right\rangle_{0}$, which can be further written as

$$
\left\langle L-L_{0}\right\rangle_{0}=-\lambda \int d \mathbf{r} \int d \Omega\left\langle e^{i \boldsymbol{\mu} \cdot \mathcal{G}(\mathbf{r})}\right\rangle_{0}-\frac{1}{2} \int d \mathbf{r}\left\langle[\mathcal{G}(\mathbf{r})+i \mathcal{F}(\mathbf{r})] \mathcal{A}^{-1}\left(\mathbf{r}-\mathbf{r}^{\prime}\right)\left[\mathcal{G}\left(\mathbf{r}^{\prime}\right)+i \mathcal{F}\left(\mathbf{r}^{\prime}\right)\right]\right\rangle_{0}
$$

The quantity $\left\langle e^{i \boldsymbol{\mu} \cdot \mathcal{G}(\mathbf{r})}\right\rangle_{0}$ can be evaluated using standard techniques for gaussian integrals. The result is

$$
\left\langle e^{i \boldsymbol{\mu} \cdot \mathcal{G}(\mathbf{r})}\right\rangle_{0}=e^{-\frac{1}{2} \boldsymbol{\mu} \cdot \mathcal{T}_{R} \cdot \boldsymbol{\mu}+\gamma(\mathbf{r}) \cdot \boldsymbol{\mu}} .
$$

The average $\left\langle[\mathcal{G}(\mathbf{r})+i \mathcal{F}(\mathbf{r})] \mathcal{A}^{-1}\left(\mathbf{r}-\mathbf{r}^{\prime}\right)\left[\mathcal{G}\left(\mathbf{r}^{\prime}\right)+i \mathcal{F}\left(\mathbf{r}^{\prime}\right)\right]\right\rangle_{0}$ can be evaluated using standard techniques for gaussian integrals as well. For simplicity, we write $\boldsymbol{\xi}(\mathbf{r})=\mathcal{G}(\mathbf{r})+i \mathcal{F}(\mathbf{r})$. Then,

$$
\begin{aligned}
\left\langle\boldsymbol{\xi}(\mathbf{r}) \mathcal{A}^{-1}\left(\mathbf{r}-\mathbf{r}^{\prime}\right) \xi\left(\mathbf{r}^{\prime}\right)\right\rangle_{0} & =\frac{1}{\Xi_{0}} \int \mathcal{D} \mathbf{P} \int \mathcal{D} \mathcal{G} \xi(\mathbf{r}) \mathcal{A}^{-1}\left(\mathbf{r}-\mathbf{r}^{\prime}\right) \xi\left(\mathbf{r}^{\prime}\right) e^{-L_{0}[\mathbf{P}, \mathcal{G}]} \\
& =\left.\frac{1}{\Xi_{0}} \frac{\delta}{i \delta \mathbf{J}(\mathbf{r})} \cdot \mathcal{A}\left(\mathbf{r}-\mathbf{r}^{\prime}\right) \cdot \frac{\partial}{i \partial \mathbf{J}\left(\mathbf{r}^{\prime}\right)}\left(\int \mathcal{D} \mathbf{P} \int \mathcal{D} \xi e^{-L_{0}[\mathbf{P}, \mathcal{G}]+i \int d \mathbf{r}_{1} \mathbf{J}\left(\mathbf{r}_{1}\right) \cdot \xi\left(\mathbf{r}_{1}\right)}\right)\right|_{\mathbf{J}=\mathbf{0}}
\end{aligned}
$$

Evaluation of the above gaussian integral gives

$$
\begin{aligned}
\int d \mathbf{r} \int d \mathbf{r}^{\prime}\left\langle\xi(\mathbf{r}) \mathcal{A}^{-1}\left(\mathbf{r}-\mathbf{r}^{\prime}\right) \xi\left(\mathbf{r}^{\prime}\right)\right\rangle_{0}= & \int \frac{d \mathbf{k}}{(2 \pi)^{3}} V \frac{\varepsilon_{0}}{\beta+\varepsilon_{0} \tilde{a}(\mathbf{k})} \operatorname{tr} \widetilde{\mathcal{T}}(\mathbf{k})-\int \frac{d \mathbf{k}}{(2 \pi)^{3}} \frac{1}{\tilde{a}(\mathbf{k})}\left[\widetilde{\mathcal{E}}_{0}(\mathbf{k})-\widetilde{\mathcal{F}}(\mathbf{k})\right] \\
& \times\left(\mathbb{1}-\frac{\varepsilon_{0}}{\beta+\varepsilon_{0} \tilde{a}(\mathbf{k})} \widetilde{\mathcal{T}}(\mathbf{k})\right)^{2}\left[\widetilde{\mathcal{E}}_{0}(-\mathbf{k})-\widetilde{\mathcal{F}}(-\mathbf{k})\right] .
\end{aligned}
$$

The variational grand potential in Eq. (16) is obtained upon substitution of the relevant terms into Eq. (13) followed by additional algebraic simplification.

\section{APPENDIX C: STATIONARY POINT OF THE VARIATIONAL GRAND POTENTIAL}

In this section, we extremize $\beta W$ in Eq. (16) with respect to the variational parameters $\tilde{a}(\mathbf{k})$ and $\widetilde{\mathcal{F}}(\mathbf{k})$. First, setting $\delta \beta W / \delta \tilde{a}(\mathbf{k})=0$, we obtain

$$
\begin{aligned}
& \frac{1}{2} V \frac{\beta^{2}}{\tilde{a}(\mathbf{k})\left[\beta+\varepsilon_{0} \tilde{a}(\mathbf{k})\right]^{2}}-\left(\frac{\varepsilon_{0}}{\beta+\varepsilon_{0} \tilde{a}(\mathbf{k})}\right)^{3}\left[\widetilde{\mathcal{E}}_{0}(\mathbf{k})-\widetilde{\mathcal{F}}(\mathbf{k})\right] \widetilde{\mathcal{T}}(\mathbf{k})\left[\widetilde{\mathcal{E}}_{0}(-\mathbf{k})-\widetilde{\mathcal{F}}(-\mathbf{k})\right] \\
& \quad=\lambda \int d \mathbf{r} \int d \Omega\left[\frac{\beta \varepsilon_{0}}{2\left(\beta+\varepsilon_{0} \tilde{a}(\mathbf{k})\right)^{2}} \boldsymbol{\mu} \cdot \widetilde{\mathcal{T}}(\mathbf{k}) \cdot \boldsymbol{\mu}-\left(\frac{\varepsilon_{0}}{\beta+\varepsilon_{0} \tilde{a}(\mathbf{k})}\right)^{2} \boldsymbol{\mu} \cdot \widetilde{\mathcal{T}}(\mathbf{k}) \cdot\left[\widetilde{\mathcal{E}}_{0}(\mathbf{k})-\widetilde{\mathcal{F}}(\mathbf{k})\right] e^{i \mathbf{k} \cdot \mathbf{r}}\right] e^{-f(\boldsymbol{\mu}, \mathbf{r})}
\end{aligned}
$$

Next, setting $\delta \beta W / \delta \widetilde{\mathcal{F}}(\mathbf{k})=\mathbf{0}$, we obtain

$$
\begin{aligned}
& \left(\frac{\varepsilon_{0}}{\beta+\varepsilon_{0} \tilde{a}(\mathbf{k})}\right) \widetilde{\mathcal{T}}(\mathbf{k})\left[\widetilde{\mathcal{E}}_{0}(\mathbf{k})-\widetilde{\mathcal{F}}(\mathbf{k})\right] \\
& =\lambda \int d \mathbf{r} \int d \Omega \widetilde{\mathcal{T}}(\mathbf{k}) \boldsymbol{\mu} e^{-f(\boldsymbol{\mu}, \mathbf{r})-i \mathbf{k} \cdot \mathbf{r}} .
\end{aligned}
$$

Substituting Eq. (C2) into Eq. (C1), we simplify Eq. (C1) to Eq. (20a). In addition, based on the uniqueness theorem in electrostatic interactions, Eq. (C2) simplifies to Eq. (20b).

\section{APPENDIX D: EVALUATION OF $\widetilde{\mathcal{F}}(k)$ IN THE LINEAR RESPONSE REGIME}

Here, we solve Eq. (26) for the variational parameter $\widetilde{\mathcal{F}}(\mathbf{k})$. For $\mathbf{k} \neq \mathbf{0}$, the matrix $\widetilde{\mathcal{T}}(\mathbf{k})$ is well defined, and therefore, we can express $\widetilde{\mathcal{F}}(\mathbf{k})$ as

$$
\widetilde{\mathcal{F}}(\mathbf{k})=-y\left(\mathbb{1}+y \frac{\varepsilon_{0}}{\beta} \widetilde{\mathcal{T}}(\mathbf{k})\right)^{-1}\left(\mathbb{1}-\frac{\varepsilon_{0}}{\beta} \widetilde{\mathcal{T}}(\mathbf{k})\right) \widetilde{\mathcal{E}}_{0}(\mathbf{k}) .
$$

The inverse operator of $\mathbb{1}+y \frac{\varepsilon_{0}}{\beta} \widetilde{\mathcal{T}}(\mathbf{k})$ can be found using a procedure similar to that presented in Eq. (B1). The procedure 
leads to

$$
\left(\mathbb{1}+y \frac{\varepsilon_{0}}{\beta} \widetilde{\mathcal{T}}(\mathbf{k})\right)^{-1}=\mathbb{1}-\frac{y}{1+y} \frac{\varepsilon_{0}}{\beta} \widetilde{\mathcal{T}}(\mathbf{k}) .
$$

Then, the evaluation of Eq. (D2) gives the expression for $\widetilde{\mathcal{F}}(\mathbf{k})$ at $\mathbf{k} \neq \mathbf{0}$,

$$
\widetilde{\mathcal{F}}(\mathbf{k})=-y\left(\mathbb{1}-\frac{\varepsilon_{0}}{\beta} \widetilde{\mathcal{T}}(\mathbf{k})\right) \widetilde{\mathcal{E}}_{0}(\mathbf{k}) \quad \text { for } \mathbf{k} \neq \mathbf{0} .
$$

For $\mathbf{k}=0, \tilde{\mathcal{T}}(\mathbf{k})$ is not well-defined in the $\mathbf{k}$-space. The expression in Eq. (26) is best solved in the position space instead. We obtain the equation in the position space by inverse Fourier transform of Eq. (26), which gives

$$
\begin{aligned}
& \int d \mathbf{r}^{\prime}\left(1 \delta\left(\mathbf{r}-\mathbf{r}^{\prime}\right)+y \frac{\varepsilon_{0}}{\beta} \mathcal{T}\left(\mathbf{r}-\mathbf{r}^{\prime}\right)\right) \mathcal{F}\left(\mathbf{r}^{\prime}\right) \\
& =-\int d \mathbf{r}^{\prime} y\left(\mathbb{1} \delta\left(\mathbf{r}-\mathbf{r}^{\prime}\right)-\frac{\varepsilon_{0}}{\beta} \mathcal{T}\left(\mathbf{r}-\mathbf{r}^{\prime}\right)\right) \mathcal{E}_{0}\left(\mathbf{r}^{\prime}\right) .
\end{aligned}
$$

The $\mathbf{k}=\mathbf{0}$ value of $\widetilde{\mathcal{E}}_{0}(\mathbf{k})$ and $\widetilde{\mathcal{F}}(\mathbf{k})$ contributes to spatially uniform fields in the $\mathbf{r}$ space. For uniform $\mathcal{E}_{0}(\mathbf{r})$ and $\mathcal{F}(\mathbf{r})$, Eq. (D4) becomes

$$
\begin{aligned}
& \int d \mathbf{r}^{\prime}\left(\mathbb{1} \delta\left(\mathbf{r}-\mathbf{r}^{\prime}\right)+y \frac{\varepsilon_{0}}{\beta} \mathcal{T}\left(\mathbf{r}-\mathbf{r}^{\prime}\right)\right) \mathcal{F} \\
& =-\int d \mathbf{r}^{\prime} y\left(\mathbb{1} \delta\left(\mathbf{r}-\mathbf{r}^{\prime}\right)-\frac{\varepsilon_{0}}{\beta} \mathcal{T}\left(\mathbf{r}-\mathbf{r}^{\prime}\right)\right) \mathcal{E}_{0}
\end{aligned}
$$

which then gives

$$
\left(1+\frac{y}{3}\right) \mathcal{F}=-y\left(1-\frac{1}{3}\right) \mathcal{E}_{0}
$$

based on the expression for $\mathbf{T}(\mathbf{r})$ in Eq. (2). Therefore, for $\mathbf{k}=\mathbf{0}$, the variational parameter $\widetilde{\mathcal{F}}(\mathbf{k})$ has stationary value given by

$$
\widetilde{\mathcal{F}}(\mathbf{k})=-\frac{2 y}{y+3} \widetilde{\mathcal{E}}_{0}(\mathbf{k}) \quad \text { for } \mathbf{k}=\mathbf{0}
$$

${ }^{1}$ R. Affleck, C. A. Haynes, and D. S. Clark, Proc. Natl. Acad. Sci. U. S. A. 89, 5167 (1992).

${ }^{2}$ M. A. Young, B. Jayaram, and D. L. Beveridge, J. Phys. Chem. B 102, 7666 (1998).

${ }^{3}$ D. Pines, S. Keinan, P. M. Kiefer, J. T. Hynes, and E. Pines, J. Phys. Chem. B 119, 9278 (2015).

${ }^{4}$ M. A. Voinov, C. T. Scheid, I. A. Kirilyuk, D. G. Trofimov, and A. I. Smirnov, J. Phys. Chem. B 121, 2443 (2017).

${ }^{5}$ E. S. Jarnikova, M. V. Parkhats, A. S. Stasheuski, and B. M. Dzhagarov, J. Appl. Spectrosc. 83, 903 (2017).

${ }^{6}$ S. Link, M. B. Mohamed, and M. A. El-Sayed, J. Phys. Chem. B 103, 3073 (1999).

${ }^{7}$ G. A. Rance and A. N. Khlobystov, Dalton Trans. 43, 7400 (2014).

${ }^{8}$ Y. Nakagawa, H. Nakazawa, and S. Kato, Langmuir 32, 6806 (2016).

${ }^{9}$ M. A. A. Fakhree, D. R. Delgado, F. Martínez, and A. Jouyban, AAPS PharmSciTech 11, 1726 (2010).

${ }^{10}$ X. Zhang, Q. Yin, P. Cui, Z. Liu, and J. Gong, Ind. Eng. Chem. Res. 51, 6933 (2012).

${ }^{11}$ H. M. Heitzer, T. J. Marks, and M. A. Ratner, Acc. Chem. Res. 49, 1614 (2016).

${ }^{12}$ H. Fröhlich, Theory of Dielectrics (Oxford University Press, London, 1958).

${ }^{13}$ M. Mandel and P. Mazur, Physica 24, 116 (1958).

${ }^{14}$ P. J. W. Debye, Phys. Z. 13, 97 (1912).

${ }^{15}$ P. J. W. Debye, Polar Molecules (Chemical Catalog Company, New York, 1929).

${ }^{16}$ L. Onsager, J. Am. Chem. Soc. 58, 1486 (1936).

${ }^{17}$ C. J. F. Böttcher, Theory of Electric Polarization, 2nd ed. (Elsevier, Amsterdam, 1973), Vol. 1.
${ }^{18}$ J. G. Kirkwood, J. Chem. Phys. 7, 911 (1939).

${ }^{19}$ M. Neumann, Mol. Phys. 50, 841 (1983).

${ }^{20}$ J. N. Wilson, Chem. Rev. 25, 377 (1939).

${ }^{21}$ D. V. Matyushov and B. M. Ladanyi, J. Chem. Phys. 110, 994 (1999).

${ }^{22}$ A. Tani, D. Henderson, J. A. Barker, and C. E. Hecht, Mol. Phys. 48, 863 (1983).

${ }^{23}$ S. Goldman and C. Joslin, J. Chem. Phys. 99, 3021 (1993).

${ }^{24}$ D. W. Jepsen, J. Chem. Phys. 45, 709 (1966).

${ }^{25}$ D. Chandler, J. Chem. Phys. 67, 1113 (1977).

${ }^{26}$ G. Nienhuis and J. M. Deutch, J. Chem. Phys. 55, 4213 (1971).

${ }^{27}$ J. S. Høye and G. Stell, J. Chem. Phys. 61, 562 (1974).

${ }^{28}$ B. U. Felderhof, G. W. Ford, and E. G. D. Cohen, J. Stat. Phys. 28, 135 (1982).

${ }^{29}$ M. S. Wertheim, J. Chem. Phys. 55, 4291 (1971).

${ }^{30}$ M. S. Wertheim, Mol. Phys. 25, 211 (1973).

${ }^{31}$ S. A. Adelman and J. M. Deutch, in Advances in Chemical Physics: NonSimple Liquids (John Wiley \& Sons, Inc., 1975), pp. 103-153.

${ }^{32}$ S. L. Carnie and G. N. Patey, Mol. Phys. 47, 1129 (1982).

${ }^{33}$ P. H. Fries and G. N. Patey, J. Chem. Phys. 82, 429 (1985).

${ }^{34}$ P. T. Cummings and L. Blum, J. Chem. Phys. 85, 6658 (1986).

${ }^{35}$ A. D. Buckingham, Aust. J. Chem. 6, 93 (1953).

${ }^{36}$ M. K. Gilson and B. H. Honig, Biopolymers 25, 2097 (1986).

${ }^{37}$ S. J. Suresh and V. M. Naik, J. Chem. Phys. 116, 4212 (2002).

${ }^{38}$ C. G. Gray, K. E. Gubbins, and C. G. Joslin, Theory of Molecular Fluids, Volume 2: Applications, International Series of Monographs on Chemistry (Oxford University Press, Oxford, UK, 2011).

${ }^{39}$ J.-P. Hansen and I. R. McDonald, Theory of Simple Liquids, 3rd ed. (Academic Press, Burlington, 2006).

${ }^{40}$ F. Schmid, J. Phys.: Condens. Matter 10, 8105 (1998).

${ }^{41}$ G. H. Fredrickson, V. Ganesan, and F. Drolet, Macromolecules 35, 16 (2002).

${ }^{42} \mathrm{G}$. H. Fredrickson, The Equilibrium Theory of Inhomogeneous Polymers (Oxford University Press, Oxford, UK, 2006).

${ }^{43}$ R. D. Coalson and A. Duncan, J. Chem. Phys. 97, 5653 (1992).

${ }^{44}$ R. D. Coalson and A. Duncan, J. Phys. Chem. 100, 2612 (1996).

${ }^{45}$ A. Abrashkin, D. Andelman, and H. Orland, Phys. Rev. Lett. 99, 077801 (2007).

${ }^{46}$ I. Nakamura, A.-C. Shi, and Z.-G. Wang, Phys. Rev. Lett. 109, 257802 (2012).

${ }^{47}$ R. Kumar, B. G. Sumpter, and M. Muthukumar, Macromolecules 47, 6491 (2014).

${ }^{48}$ A. Levy, D. Andelman, and H. Orland, Phys. Rev. Lett. 108, 227801 (2012).

${ }^{49}$ A. Levy, D. Andelman, and H. Orland, J. Chem. Phys. 139, 164909 (2013).

${ }^{50}$ J. M. Martin, W. Li, K. T. Delaney, and G. H. Fredrickson, J. Chem. Phys. 145, 154104 (2016).

${ }^{51}$ Z.-G. Wang, Phys. Rev. E 81, 021501 (2010).

${ }^{52}$ R. R. Netz and H. Orland, Eur. Phys. J. E 11, 301 (2003).

${ }^{53}$ C. Caleman, P. J. van Maaren, M. Hong, J. S. Hub, L. T. Costa, and D. van der Spoel, J. Chem. Theory Comput. 8, 61 (2012).

${ }^{54}$ J. D. Ramshaw, J. Chem. Phys. 55, 1763 (1971).

${ }^{55}$ O. D. Kellogg, Foundations of Potential Theory (Springer Berlin Heidelberg, 1967).

${ }^{56}$ J. D. Ramshaw, J. Chem. Phys. 70, 1577 (1979).

${ }^{57}$ I. Borukhov, D. Andelman, and H. Orland, Phys. Rev. Lett. 79, 435 (1997).

${ }^{58}$ S. Qi, H. Behringer, and F. Schmid, New J. Phys. 15, 125009 (2013).

${ }^{59}$ R. P. Feynman, Statistical Mechanics: A Set of Lectures (W. A. Benjamin, Inc., Reading, Massachusetts, 1972).

${ }^{60}$ Strictly speaking, the Gibbs-Feynman-Bogoliubov inequality in Eq. (13) only holds when the actions are real. In our case, the actions can take complex values and, thus, the inequality is not always true. The true (real) value of the upper bound of $W$ is approached in an oscillatory manner in the complex function space. Nevertheless, one may show that the stationary point is the upper bound of the grand potential, and the result of the variational approach remains valid. We refer interested readers to Ref. 79 for more details.

${ }^{61}$ P. T. Leung and G. J. Ni, Eur. J. Phys. 27, N1 (2006).

${ }^{62}$ J. D. Jackson, Classical Electrodynamics, 2nd ed. (Wiley, New York, 1998).

${ }^{63}$ See Sec. 4.5 and Eq. (4.65) of Ref. 62, for example.

${ }^{64} \mathrm{To}$ second order in the field $\bar{\phi}$ in the exponent, Eq. (14) in Ref. 50 can be written as $Q=\frac{1}{V} \int d \mathbf{r} \exp \left[-i \bar{w}(\mathbf{r})-i q \bar{\phi}(\mathbf{r})-\frac{\bar{\mu}^{2}}{6}|\nabla \bar{\phi}(\mathbf{r})|^{2}\right]$. 
${ }^{65}$ R. A. DiStasio, Jr., V. V. Gobre, and A. Tkatchenko, J. Phys.: Condens. Matter 26, 213202 (2014).

${ }^{66}$ D. J. Grzetic, K. T. Delaney, and G. H. Fredrickson, J. Chem. Phys. 148, 204903 (2018).

${ }^{67}$ H. Kleinert, Path Integrals in Quantum Mechanics, Statistics, Polymer Physics, and Financial Markets (World Scientific, Singapore, 2009).

${ }^{68}$ S. F. Edwards, Proc. Phys. Soc. 85, 613 (1965).

${ }^{69}$ K. F. Freed, J. Chem. Phys. 55, 3910 (1971).

${ }^{70}$ M. W. Matsen and M. Schick, Macromolecules 27, 6761 (1994).

${ }^{71}$ G. H. Fredrickson and E. Helfand, J. Chem. Phys. 87, 697 (1987).

${ }^{72}$ J. Qin and D. C. Morse, Phys. Rev. Lett. 108, 238301 (2012).
${ }^{73}$ A. Alexander-Katz, A. G. Moreira, S. W. Sides, and G. H. Fredrickson, J. Chem. Phys. 122, 014904 (2004).

${ }^{74}$ J.-L. Barrat, G. H. Fredrickson, and S. W. Sides, J. Phys. Chem. B 109, 6694 (2005).

${ }^{75}$ A. Alexander-Katz and G. H. Fredrickson, Macromolecules 40, 4075 (2007).

${ }^{76}$ J. Lee, Y. O. Popov, and G. H. Fredrickson, J. Chem. Phys. 128, 224908 (2008).

${ }^{77}$ R. Wang and Z.-G. Wang, J. Chem. Phys. 142, 104705 (2015).

${ }^{78}$ M. Kardar, Statistical Physics of Fields (Cambridge University Press, Cambridge, UK, 2007).

${ }^{79}$ D. Frydel, Eur. J. Phys. 36, 065050 (2015). 